\title{
El sistema de evaluación global (seg) como instrumento de valoración de la veracidad del testimonio
}

\section{The global evaluation system (seg) as an instrument for assessing the veracity of the testimony}

DOI: $10.46932 / \mathrm{sjjdv2n3-053}$

Received in: May 1st, 2021

Accepted in: Jun 30th, 2021

\author{
Christian Gustavo Mena Ávila \\ Psicólogo Clínico \\ Institución actual: Ministerio de Salud Pública del Ecuador \\ Dirección completa: Ecuador, Azuay, Cuenca \\ Correo electrónico: Chrisgustavo@ hotmail.com
}

\begin{abstract}
RESUMEN
Para el ser humano ha sido de mucho valor poder impartir justicia basándose en los hechos de un acontecimiento y los testimonios que de él recibe, por lo tanto, poder determinar la veracidad de los testimonios de los testigos, las víctimas y los agresores, y así poder obtener conclusiones apegadas a la realidad e impartir la justicia que anhela, esto ha sido objeto de numerosos estudios.

Lo que busca este artículo es ir detallando el avance científico-histórico de la toma de testimonios y fundamentar en qué criterios se basan para determinar si un testimonio es creíble, no creíble o indeterminado, todo esto en base al Sistema de Evaluación Global (SEG), y para eso se realizó una revisión sistémica de diferentes artículos en la base de datos de Scopus, Web of Sciencie y Google Scholar entre las palabras clave que se usaron están: deception detection, detection of the lie, Sistema de evaluación Global, seleccionando 25 artículos publicados entre los años 2005 y 2021
\end{abstract}

Palabras clave: Sistema de evaluación global, detección del engaño, detección de la mentira, psicología de la mentira.

\begin{abstract}
For the human being it has been of great value to be able to impart justice based on the facts of an event and the testimonies he receives from it, therefore, to be able to determine the veracity of the testimonies of witnesses, victims and aggressors, and thus be able to obtain conclusions attached to reality and impart the justice he longs for, this has been the subject of numerous studies.

The aim of this article is to detail the scientific-historical progress of the taking of testimonies and to establish on what criteria are based to determine whether a testimony is credible, not credible or indeterminate, all this based on the Global Evaluation System (SEG), and for that a systemic review of different articles in the database of Scopus, Web of Sciencie and Google Scholar among the keywords used are: deception detection, detection of the lie, Global Evaluation System, selecting 25 articles published between the years 2005 and 2021.
\end{abstract}

Keywords: Global evaluation system, deception detection, detection of the lie, psychology of the lie. 


\section{INTRODUCCIÓN}

A través del tiempo el ser humano ha intentado y estructurado diferentes métodos para obtener la verdad en un testimonio, desde el uso del miedo, la tortura y la confrontación física que se usaba en la época antigua especialmente en Grecia Y Roma y otros métodos que involucraban la protección divina.

Luego de toda esta época apareció por alrededor del año 2010 la psicología del testimonio en donde los conocimientos de los procesos psicológicos como la memoria la atención y la percepción en la recolección de los testimonios recobraron importancia útil, posteriormente y gracias al avance de la tecnología nuevos elementos se fueron incorporando al análisis de la mentira como es el caso de los métodos en base a la activación de zonas cerebrales por ejemplo un tomógrafo de resonancia nuclear que a través de un campo magnético mide la intensidad de irrigación de distintas zonas cerebrales que simplemente demostró que no existe ninguna zona del cerebro especializada en la mentira. necesario también mencionar el famoso polígrafo inventado en 1921 por John Larson que registra actividad respiratoria, presión arterial y actividad cardiovascular y la actividad galvánica de la piel a través de las glándulas sudoríparas. (Medina,2019).

En los últimos tiempos, se ha involucrado en el tema del engaño más opciones a los protocolos que se basan en la ansiedad y que postulan que hay una carga cognitiva en la mentira, en la formulación de preguntas. (Vrij et al., 2012).

A lo anteriormente mencionado se suma que existen pruebas de que mentir es cognitivamente más complicado que decir la verdad y por ende un proceso mucho más complicado de análisis (Vrij et al., 2006).

Sin embargo, en la actualidad y a pesar de toda la tecnología que existe, los estudios de neuroimagen que tratan de la detección del engaño se ven empañados por la ausencia de claridad en sus conceptos y una serie de problemas que no son únicos de la neuroimagen (Meijer et al.,2016).

\section{METODOLOGÍA}

Se realizó una revisión sistemática de los artículos que se encuentran en las bases de datos científicas digitales Scopus, Web of Science y Google Schollar, a las que se accedió a través de la Biblioteca Virtual de la Universidad Católica de Cuenca. Como criterios de inclusión de los artículos se establecieron: a) haber sido publicados durante los últimos 5 años, en lo que a Scopus Y Web Of Science se refiere a diferencia de Google Schollar que se tuvo que ampliar la búsqueda desde el año 2006 para obtener información importante para esta revisión b) estar redactados en inglés o español y, c) ser revisiones sistemáticas o metaanálisis. Como criterios de exclusión está: a) ser de acceso restringido, b) ser libros o capítulos de libros, c) abordar temas diferentes a la detección del engaño, la mentira o el 
Sistema de Evaluación Global. Se consideraron 25 artículos, utilizando para su selección el sistema PRISMA (Preferred Reporting Items for Systematic reviews and Meta-Analyses), que se verifica en la Figura 1.

Número de registros o citas identificadas en las búsquedas:

- Scopus: $(n=85)$

- Web of Science: $(n=40)$

- Google Schollar $(\mathrm{n}=18)$

Total de registros: $(n=143)$

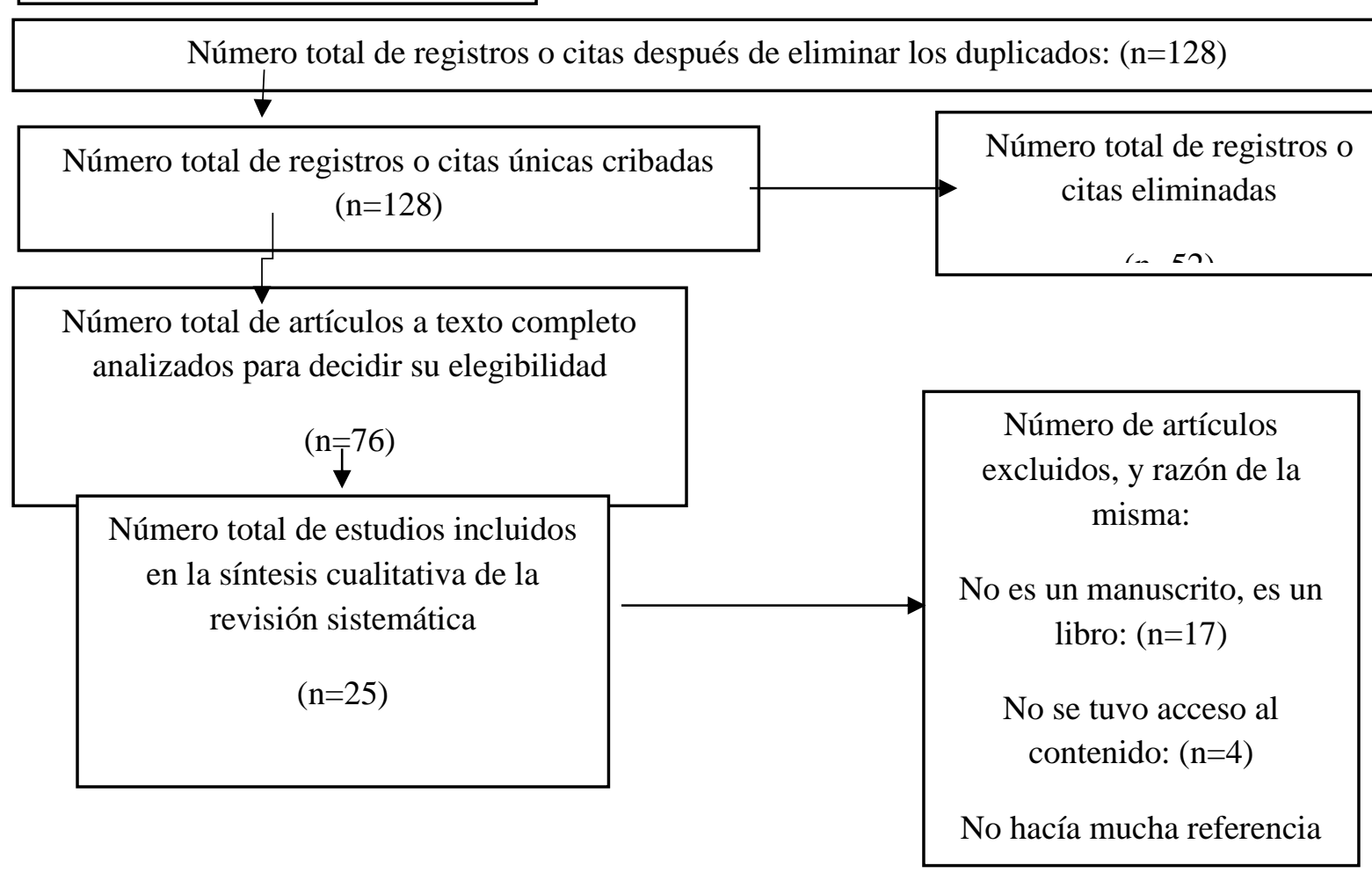

\section{DETECCIÓN DEL ENGAÑO A TRAVÉS DEL TIEMPO}

En cuanto a la forma de determinar el engaño en las personas hay variedad de enfoques desde el lingüístico hasta las que implican tecnología avanzada y/o procesos complejos de interrogatorio, en cuanto a la investigación del engaño verbal ha confirmado que la cantidad total de información proporcionada es una de las señales más diagnósticos del engaño, ya que los que dicen la verdad suelen informar con más detalles que los mentirosos (Amado et al., 2015)

En un experimento diseñado por (Vrij et al., 2018) Encontró que tanto los que dicen la verdad como los que mienten agregan detalles a sus narraciones, y como, el número total de detalles no se diferencia entre los dos. En ese sentido cobra importancia el tipo de detalles que ampliaron que fue 
diferente. A oposición de los mentirosos, los que dicen la verdad agregaron más a sus cuentos y hacían que sus narraciones estuvieran menos guionizadas.

(Klaver, et al., 2007) en un experimento con 45 delincuentes masculinos evidenció que los rasgos de la psicopatía eran asociados con dictámenes desfavorables sobre la capacidad de mentir, la verborrea y el aumento del parpadeo. Mientras mentían, los delincuentes más psicopáticos se expresaban de forma más rápida y evidenciaron aumentos en el parpadeo y en los movimientos de la cabeza. Los indicadores de engaño en los delincuentes eran diferentes de los que se observan en las poblaciones de no delincuentes lo que contribuyó al estudio del engaño en la actualidad.

En un meta análisis realizado por (Sternglanz et al., 2019). sobre una variedad de temas relacionados con la detección del engaño; y para un breve resumen se concluye que estos análisis indican que, a nivel general, las personas son ligeramente mejores que el azar en la detección del engaño, independientemente de sus rasgos de personalidad, experiencia profesional o la seguridad en sus juicios. Hay sospechas que indican de forma probabilística cuándo la gente puede estar mintiendo, pero sólo minoría de mentirosos muestran indicios claros. La restringida exactitud en la detección del engaño puede imputarse principalmente al hecho de que los indicios válidos de engaño no son muy confiables. Sin embargo, los programas de adiestramiento que se centralizan en pistas documentadas del engaño, el contenido verbal y la práctica pueden mejorar la capacidad de los receptores para detectar un poco mejor el engaño. Además, existen los programas informáticos y algoritmos, que son una enorme ayuda en la detección de la mentira.

La tendencia de los mentirosos para crear historias y retratar escenarios falsos con el aumento de detalles, con falsas percepciones y manipulando el contexto, han hecho que se desarrollen numerosos métodos de detección del engaño (Nahari,2018).

Por otro lado, los que dicen la verdad suelen dar más detalles que los mentirosos y a la final "mentir es cognitivamente más exigente que nuestro comportamiento por defecto de decir simplemente la verdad" (Blandon et al, 2014) (Walczyk, et al, 2013).

Vale la pena destacar que los interrogativos severos son ineficientes a la hora de obtener un testimonio verdadero, primero porque aumenta la resistencia del sujeto, en segundo lugar, por el carácter amenazante de este tipo de interrogatorios que va en contra del objetivo de recuperar información valiosa en el testimonio y reduce la probabilidad de obtener información extensa. (Vrij, et al., 2017). 


\section{DESARROLLO DE PROTOCOLOS PARA LA DETECCIÓN DEL ENGAÑO ORIENTACIÓN NO VERBAL-EMOCIONAL EN LA DETECCIÓN DE LAS MENTIRAS}

Básicamente la teoría nos dice que la conducta no verbal es muy diferente a la conducta que la persona muestra cuando dice la verdad, la persona puede vivenciar los siguientes procesos: a) activación psicofisiológica, b) emociones como culpa o miedo de ser descubierto c) dificultad en los procesos cognitivos para elaborar una mentira y d) controlar su conducta para evitar dar señales (Blandón et al.,2017)..

Al mentir la persona tiende a sentir emociones que tiene que disimular o enmascarar, esto nos manifiesta de que los factores emocionales alteran los procesos cognitivos y a su ves se ve reflejado en la parte conductual

\section{ENTREVISTA COGNITIVA MEJORADA}

Se encuentra entre las técnicas más eficaces a la hora de obtener declaraciones y de minimizar la mentira; ésta se divide en cinco momentos claves (en un inicio era cuatro): a) La reinstauración del contexto en donde su principio es que dl recobro de información de la memoria es mucho mejor cuando se recrea el contexto de lo sucedido de forma original durante el interrogatorio. Aquí el interrogador solicita al testigo que recree en su mente la escena de la transgresión y su estado psicológico, cognitivo y emocional. B) La técnica de contarlo todo radica en solicitar al testigo que comente todo lo que recuerde sobre el crimen, aunque sean detalles que en un principio sean considerados sin importancia. La técnica del cambio de perspectiva reside en pedir al testigo que se acuerde del acontecimiento desde una representación diferente. Por ejemplo, si el testigo está muy perturbado, pedirle que conmemore desde una representación más tranquila, finalmente, la técnica de cambio de orden consiste en pedir al declarante que cuente los sucesos en un orden diferente al cronológico, por ejemplo, iniciando desde el final. (Paulo et al., 2014)

\section{CBCA (ANÁLISIS DEL CONTENIDO BASADO EN CRITERIOS)}

Se trata de una amplia gama de criterios de la realidad que se agrupan en cognitivos y motivacionales; los cognitivos se basan en la facilidad que tiene un narrador de contar un acontecimiento desde la memoria episódica, mientras que los que mienten construyen el relato desde la memoria semántica, de la misma manera los motivacionales son aquellos que intentan convencer al oyente.

En el CBCA originalmente se habla de 19 criterios de la realidad que aparecen con mayor intensidad que aparecen con mayor énfasis en las situaciones experimentadas que en los inventados y estos criterios son: 
1. Estructura lógica

2. Producción no estructurada

3. Cantidad de detalles

4. Incorporación contextual

5. Descripciones de las interacciones

6. Reproducción de la conversación

7. Complicaciones inesperadas durante el incidente

8. Detalles inusuales

9. Detalles superfluos

10. Detalles informados con precisión y malinterpretados

11. Asociaciones externas relacionadas

12. Relatos del estado mental subjetivo

13. Atribución del estado mental del agresor

Contenidos relacionados con la motivación

14. Correcciones espontáneas

15. Admitir la falta de memoria

16. Plantear dudas sobre el propio testimonio

17. Autodesprecio

18. Perdonar al autor del delito

Elementos Específicos

19. Detalles característicos del evento

Todos estos 19 criterios se analizan para determinar de alguna manera la realidad o veracidad de un testimonio. (Sporer et al.,2021)

\section{PROTOCOLO SVA}

El Sistema de Análisis de la Validez de las declaraciones (SVA) es un instrumento psicométrico, utilizado en España en el ámbito forense, y el que evalúa la credibilidad de los testimonios de los menores abusados sexualmente, analizando el contenido de sus relatos.

Se compone de tres elementos:

1. La entrevista semiestructurada con la víctima.

2. El análisis del contenido de la entrevista según determinados criterios (CBCA)

3. Por último, la integración del CBCA y los criterios correspondientes al Listado de Validez. 
En la entrevista estructurada el protocolo se realiza una entrevista delineada para conseguir la máxima información posible del testigo y poder emplear los criterios del CBCA y, a la vez, menguar cualquier tipo de corrupción generada, ya sea por el entrevistador o por cualquier otro adulto.

Se realiza una hipótesis principal y alternativas en donde se analiza: estructura lógica de los detalles, si existe elaboración desestructurada, la cantidad de los detalles, Engranaje contextual, Descripción de interacciones: acciones y reacciones mutuas entre el testigo y el agresor, Complicaciones inesperadas, Peculiaridades del contenido, detalles inusuales, detalles extraños, pero no irreales, detalles superfluos, detalles periféricos, Incomprensión de, Asociaciones externas relacionadas. (Medina et al., 2014).

\section{ENTREVISTA POR GRUPOS}

Un apartado importante es el tema de la adaptación del registro de testimonios para grupos y que esto permita el desarrollo de protocolos específicos para ellos analizando la consistencia y coherencia de los relatos que los interrogados realizan en la presentación de los hechos y así obtener una visión unificada de las diferentes fuentes de información (Vernham et al., 2016)

\section{EL SISTEMA DE EVALUACIÓN GLOBAL}

Este sistema fu adaptado al sistema jurídico español para calificar la veracidad del testimonio y la huella psíquica (Álvarez-Bello, 2013).

Por mucho tiempo se ha dejado el impacto psicológico a un lado hasta que relativamente hace poco, se desarrollaron sistemas de evaluación del testimonio para evaluar los daños psíquicos de la víctima, y esto se inició con los casos de abuso sexual que la policía registraba (Arce et al., 2014).

Arce y Fariña (2010) desarrollaron un protocolo clínico forense, el SEG (Sistema de evaluación Global) que se estructura en 9 fases:

a) Obtención de la declaración. - donde se usa la entrevista cognitiva mejorada sea en población de niños, jóvenes o discapacitados y la entrevista forense a discapacitados y para la evaluación de la huella psíquica utiliza técnicas psicométricas y la Entrevista clínica forense.

b) Repetición de la obtención de la información. que tiene como objetivo el estudio de la consistencia temporal del testimonio.

c) Contraste de las declaraciones hechas a lo largo del proceso judicial. mediante un estudio de validez SVA (SISTEMA DE ANÁLISIS DE VALIDEZ DE LAS DECLARACIONES) se analiza las otras declaraciones e interrogatorios. 
d) Análisis del contenido de las declaraciones. - donde se utiliza el procedimiento SVA/CBCA (Análisis del Contenido Basado en Criterios).

e) Análisis de la fiabilidad de las medidas. - en esta fase es indispensable un evaluador entrenado, efectivo y consistente, para las medidas de las consecuencias clínicas del hecho traumático para la detección de la huella psicológica y dilucidar si los testimonios son productos de simulación por lo que se apoya con métodos como el MMPI 2 o la entrevista clínica en formato de discurso libre.

f) Medida de las consecuencias clínicas del hecho traumático. - en esta fase se detecta la huella psíquica del delito y el hallazgo de cuadros clínicos y la determinación de que no sea producto de simulación.

g) Evaluación de la declaración de los actores implicados. - es cuando se aplica el mismo procedimiento al agresor para obtener una estimación de la validación convergente de datos.

h) Análisis de la personalidad de los actores implicados. - en este apartado se puede encontrar las claves explicativas de la agresión y/o enfermedad mental, en donde el instrumento del MMPI 2 y la entrevista clínica en formato libre para poner de manifiesto si existe o no simulación en el interrogado.

i) Implicación para la presentación de informes. - aquí el informe considera:

1) Probablemente cierto. 2) Probablemente no cierto. 3) Indeterminada.

A todo esto, hay que mencionar que la fiabilidad recae en el entrevistador/evaluador. (Arce et al., 2005)

\section{LA HUELLA PSÍQUICA EN EL SISTEMA DE EVALUACIÓN GLOBAL}

La huella psíquica es un importante elemento a analizar en las personas en las que se les haya vulnerado los derechos como por ejemplo en casos de cualquier tipo de violencia, sea esta física, sexual o psicológica que pueden desencadenar en la víctima un trastorno por Estrés Postraumático; al mismo tiempo es importante no caer en un error de diagnóstico por simulación de la víctima, en resumen en la práctica para la evidencia no solo hay que encontrar el daño emocional sino que es importante demostrarlo. (Arce y Novo, 2012).

La víctima puede presentar 5 tipos de lesiones: física, mental, sufrimiento emocional, afectación a sus derechos y pérdida o daño material.

La huella psíquica se obtiene a través de la medida de los efectos de suceso estresante con síntomas que van desde la depresión, ansiedad, molestias somáticas, inadaptación social y problemas de conducta. (Arce y Fariña, 2009) 


\section{CONCLUSIONES}

En la comunidad científica existe bastante aceptación del Sistema de Evaluación Global no solo en lo que a psicología se refiere sino también en el área legal, aunque hay que considerar el porcentaje de error que tiene la prueba, la existencia de un control sostenido por otros expertos para la revisión de pares, y un consenso general en cuanto a la validez de la teoría en la comunidad científica. (Nazaret, et al., 2020).

Es un método efectivo para determinar la huella psíquica y la simulación por su estructura que analiza diferentes situaciones que se pueden presentar durante el testimonio de la víctima; además de contar instrumentos clínicos como la entrevista cognitiva mejorada y la aplicación del MMPI-2 que hacen que su eficiencia sea alta; al mismo tiempo es importante que los que apliquen este modelo sean entrenados exhaustivamente y que tengan una alta capacidad de objetividad y evitar la subjetividad en los procesos de toma de testimonios. (Arce y Fariña., 2014).

Lo que hace muy eficiente a este sistema de toma de testimonio es que tiene una estructura adecuada para la toma de testimonios filtrada por criterios de la realidad y se basa en la objetividad y la habilidad y entrenamiento de los profesionales que realizan el interrogatorio.

Es importante destacar que todo proceso de obtención de la información puede ser mejorado mediante investigaciones que busquen como meta, brindar más objetividad al proceso y potenciando las habilidades de los interrogadores, evitando que prejuicios intervengan en el proceso de recolección de información.

El sistema de evaluación global se ha extendido a mas campos del que en un origen tuvo como el hecho del abuso sexual, se ha ampliado a temáticas como violencia de género, Mobbing, cobro de seguros médicos, entre otros, que a su vez generan un reto para el propio Sistema de Evaluación global, pues tiene que adaptarse y de cierta manera especializarse en cada temática en lo que se lo deseé aplicar.

\section{REFERENCIAS}

Álvarez-Bello, F. J. (2013). El control del engaño en la evaluación psicológica forense de la violencia de género: posibilidades y limitaciones en el contexto chileno. Anuario de psicología jurídica, 23(1), 53-60.

Amado, B. G., Arce, R., \& Fari na, F. (2015). Undeutsch hypothesis and Criteria Based Content Analysis: A meta-analytic review. The European Journal of Psychology la 
Arce, R. (2010). El Sistema de Evaluación Global en casos de violencia de género: Huella psíquica y testimonio. Informaciò Psicológica, 99, 19-35.

Arce, R., \& Novo, M. (2012). La prueba psicológica-forense del maltrato: El Sistema de Evaluación Global. Violencia de género. Módulo II: El estudio de la violencia de género: teorías explicativas, factores de riesgo y evaluación, 119-143.

Arce, R., \& Fariña, F. (2009). Evaluación psicológica forense de la credibilidad y daño psíquico en casos de violencia de género mediante el sistema de evaluación global. Violencia de género. Tratado psicológico y legal, 147-168.

Arce, R., \& Fariña, F. (2014). Práctica de la prueba psicológica-forense: El Sistema de Evaluación Global. Psychotherapy, 55, 556-570.

Arce, R., \& Fariña, F. (2014). Evaluación psicológico-forense de la credibilidad y daño psíquico mediante el Sistema de Evaluación Global. V iolencia de género: perspectiva multidisciplinar y práctica forense.

Arce, R., \& Fariña, F. (2005). Peritación psicológica de la credibilidad del testimonio, la huella psíquica y la simulación: el sistema de evaluación global (SEG). Papeles del psicólogo, 26(92), 59-77.

Blandon-Gitlin, I., Fenn, E., Masip, J., \& Yoo, A. H. (2014). Cognitive-load approaches to detect deception: Searching for cognitive mechanisms. Trends in Cognitive Sciences, 18, 441-444. https://doi.org/10.1016/j.tics.2014.05.004

Blandón-Gitlin, I., López, R. M., Masip, J., \& Fenn, E. (2017). Cognición, emoción y mentira: implicaciones para detectar el engaño. Anuario de Psicología Jurídica, 27(1), 95-106.

Klaver, JR, Lee, Z. y Hart, SD (2007). Psicopatía e indicadores no verbales de engaño en delincuentes. Ley y comportamiento humano, 31 (4), 337-351

Medina, C., \& Gómez, L. C. (2019). Detección de la mentira y credibilidad del testimonio: una revisión histórica hasta la actualidad de técnicas, instrumentos Y protocolos más utilizados. Skopein: La justicia en manos de la Ciencia, (20), 2.

Medina, J., Soriano, L., \& Negre, M. (2014). Sistema de Análisis de Validez de las Declaraciones (Protocolo SVA) en un caso de abusos sexuales entre menores. Descripción de criterios y su aplicación. Gaceta Internacional de Ciencias Forenses, 12, 69-79.

Meijer, E. H., Verschuere, B., Gamer, M., Merckelbach, H., \& Ben-Shakhar, G. (2016). Deception detection with behavioral, autonomic, and neural measures: Conceptual and methodological considerations that warrant modesty. Psychophysiology, 53(5), 593-604.

Nahari, G. (2018). Reality monitoring in the forensic context: Digging deeper into the speech of liars. Journal of Applied Research in Memory and Cognition, 7(3), 432-440.

Nazaret Martínez Mollinedo y Ma Jesús Orihuela Canto. (2020) "Aplicación del sistema de evaluación global en la valoración de la credibilidad en adultos". Almoraima. Revista de Estudios Campogibratareños, 44. Algeciras: Instituto de Estudios Campogibraltareños. 
Paulo, R. M. M., Luna, K., \& Albuquerque, P. B. (2014). La entrevista cognitiva mejorada: Cómo interrogar a un testigo de manera eficaz.

Sternglanz, R. W., Morris, W. L., Morrow, M., \& Braverman, J. (2019). A review of meta-analyses about deception detection. The Palgrave handbook of deceptive communication, 303-326.

Sporer, S. L., Manzanero, A. L., \& Masip, J. (2021). Optimizing CBCA and RM research: recommendations for analyzing and reporting data on content cues to deception. Psychology, Crime \& Law, 27(1), 1-39.

Vrij, A., \& Granhag, P. A. (2012). Eliciting cues to deception and truth: What matters are the questions asked. Journal of Applied Research in Memory and Cognition, 1(2), 110-117.

Vrij, A., Fisher, R., Mann, S., \& Leal, S. (2006). Detecting deception by manipulating cognitive load. Trends in cognitive sciences.

Vrij, A., Leal, S., Jupe, L. y Harvey, A. (2018). Medidas de detección de mentiras verbales dentro de los sujetos: una comparación entre el detalle total y la proporción de complicaciones. Psicología legal y criminológica, 23 (2), 265-279.

Vrij, A., Meissner, C. A., Fisher, R. P., Kassin, S. M., Morgan III, C. A., \& Kleinman, S. M. (2017). Psychological perspectives on interrogation. Perspectives on Psychological Science, 12(6), 927-955.

Vernham, Z., Granhag, P. A., \& Mac Giolla, E. (2016). Detecting deception within small groups: A literature review. Frontiers in psychology, 7, 1012.

Walczyk, J. J., Igou, F. P., Dixon, A. P., \& Tcholakian, T. (2013). Advancing lie detection by inducing cognitive load on liars: A review of relevant theories and techniques guided by lessons from polygraphbased approaches. Frontiers in Psychology, 4, 14. https://doi.org/10.3389/fpsyg.2013.00014 